(C) 1980. The Genetical Society of Great Britain

\title{
X-CHROMOSOME INVOLVEMENT IN MALE HYBRID STERILITY FROM GLOSSINA MORSITANS SUB-SPECIES CROSSES
}

\author{
C. F. CURTIS, P. A. LANGLEY*, and M. A. TREWERN* \\ Ross Institute, London School of Hygiene \& Tropical Medicine, London WC1E 7HT, U.K. \\ Received 19.v.80
}

\section{SUMMARY}

Crosses between sub-species of the tsetse fly Glossina morsitans yield males which can mate but are unable to inseminate. To test the hypothesis of $\mathrm{X}$ chromosome involvement in this form of male sterility, G. m. morsitans carrying the X-chromosome marker ocra were mated to wild type $G$. m. centralis, and the $F_{1}$ females backcrossed to each parental population. The ocra gene was used to distinguish those male progeny which had inherited their X-chromosomes from each sub-species and these categories of male from each backcross differed significantly in the proportion which could inseminate. It is concluded that inability to inseminate is caused by an interaction of the $\mathrm{X}$-chromosome from one sub-species with the Y-chromosome and/or autosomal factors from the other. The results are discussed in relation to similar observations from crosses within two Anopheles species complexes and to the genetic control of tsetse flies.

\section{INTRODUCTION}

GLOSSINA MORSITANS tsetse flies are the main vectors of nagana (trypanosomiasis of domestic animals) in African savannas. Several subspecies of $G$. morsitans were recognised on morphological grounds by Vanderplank (1949) and were re-named by Machado (1970). Crosses between different sub-species and between these and $G$. swynnertoni were shown to be partly sterile and to produce sterile male hybrids (Potts, 1944; Vanderplank, 1944; Vanderplank, 1948). Mating competitiveness was tested in the field (Jackson, 1945) and the first-ever field trial of a genetic control system was carried out between 1944 and 1946 using the sterility from species crosses (Vanderplank, 1947 and personal communication).

The genetics of the sterility produced by crossing $G$. morsitans subspecies was studied with more reliable laboratory rearing techniques (Curtis, 1972). It was concluded that the partial sterility in the crosses was probably due to incompatibility between the genes of pregnant females and their hybrid larvae in utero. The male hybrid sterility from all possible reciprocal crosses between three sub-species appeared to be due to a sperm defect which prevented normal migration of sperm from the spermatophore to the spermathecae in the mates of hybrid males. The ability or inability to inseminate was characteristic of an individual in repeated tests and this character segregated in the progenies of backcrosses of female hybrids to either parent. It was suggested that the sterility might be due to heterozygosity at an autosomal locus. In an analogous case in the Anopheles maculipennis complex an explanation in terms of incompatibility of alien Xand Y-chromosomes was preferred (Fraccaro et al., 1977). Involvement of

* Tsetse Research Laboratory, University of Bristol School of Veterinary Science, Langford, Bristol BS18 7DU, U.K. 
the X-chromosome in causation of the male hybrid sterility in the $A n$. gambiae complex was proved using an X-chromosome marker (Curtis, $1978 a$ and $b$ ).

An X-chromosome marker, ocra, has become available in $G$. morsitans (Bolland et al., 1974). This paper describes a test for X-chromosome involvement in causing the male hybrid sterility in this group.

\section{MAterials AND METHODS}

The stocks used were:

G. morsitans morsitans originating from Kariba, Zimbabwe, and carrying the sex linked recessive gene ocra, causing yellowish body colour, selected from within a laboratory stock by Bolland et al. (1974). The mutant stock is now maintained at Langford.

G. $m$. centralis originating from Singida, Tanzania, were supplied by I.L.R.A.D. in 1979 and maintained at Langford. The stock has wild type (dark brown) body colour.

The stocks and crossed females were maintained by feeding through artificial membranes (Mews et al., 1977). Crosses were made by isolating virgin females within 24 hours of emergence and mating them on day three to males aged at least seven days. The mated females were maintained for 90 days and the survival and number of pupae produced was recorded daily from day 16 of life so that fecundity could be calculated in terms of total pupae per aggregate number of mature female days. Each cross was made with a total of 120 females ( 15 in each of 8 cages). Each backcross was made with 75 females, 15 in each of 5 cages.

The ability of male progeny to inseminate was tested by confining them at the age of seven days with three day old $G$. m. morsitans virgin females and, after $24 \mathrm{hs}$, dissecting the females and examining the spermathecae at $280 \times$ magnification for the presence of sperm.

\section{RESUlts}

The results are summarized in table 1 and fig. 1 . The two reciprocal crosses between $G$. m. centralis and $G$. m. morsitans (ocra) showed a marked difference in fecundity, the cross with $G$. m. morsitans as the female parent being much the more fecund. The morsitans females were also more fecund than the centralis females in the within-subspecies matings and it seemed best to use the morsitans $\times$ morsitans mating as the control for the between sub-species cross where morsitans was the female parent and the centralis $\times$ centralis mating as control for the reciprocal. On this basis fecundity was reduced to 89 per cent of control in the morsitans $q \times$ centralis $\delta$ cross and to 28 per cent of control in the reciprocal cross. The eclosion rates and weights of hybrid pupae were similar to those of controls (table 1 ). The $F_{1}$ males from both the reciprocal crosses mated readily but of those tested none was able to inseminate, i.e., to introduce sperm into the spermathecae of their mates. Only the cross with $G$. $m$. morsitans as the female parent yielded sufficient progeny for an adequate backcrossing test. Samples of $F_{1}$ females from this cross were backcrossed to each of the parental stocks and the fecundity of both backcrosses is shown in table 1. The ocra and wild type male progeny were separated and the inseminating ability of each was tested 
NOTES AND COMMENTS

突苋

안픔

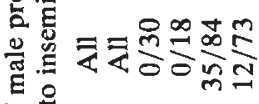

ठ

z ते

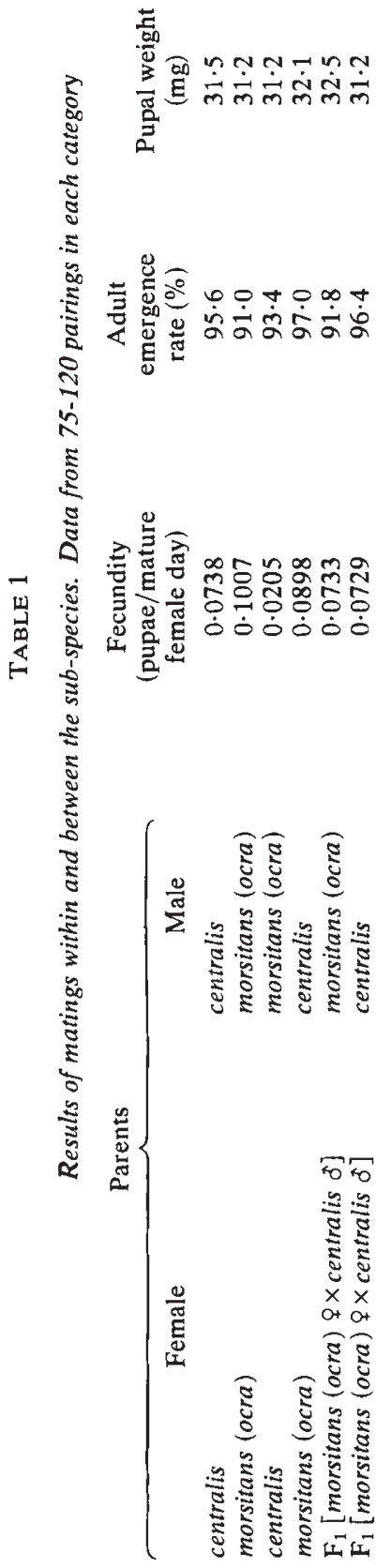




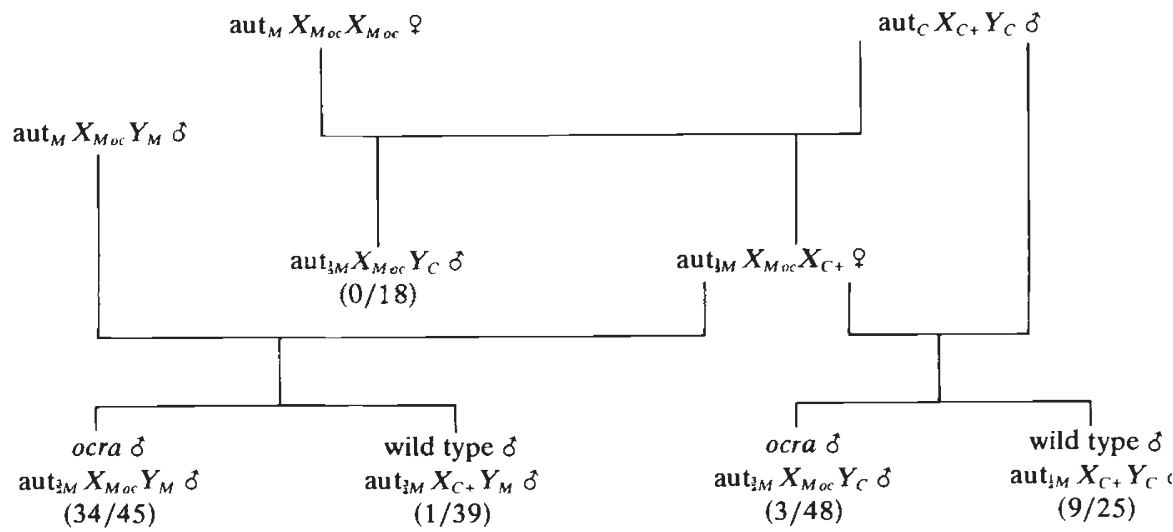

FIG. 1.-The morsitans (ocra) $q \times$ centralis $\delta$ cross and male progeny of both backcrosses of the $F_{1}$ females. The fractions in parentheses indicate the number of males which were able to inseminate/total number tested. The chromosomal compositions of each type are indicated (subscript $M$ and $C$ indicate morsitans or centralis origin of the chromosome(s), $o c=o c r a$ gene and $+=$ its wild type homologue, aut $=$ autosomes whose average composition, with respect to sub-species origin, is indicated.

(fig. 1). From the backcross to morsitans (ocra) males, the majority of the ocra progeny could inseminate ( 76 per cent) but almost none ( 3 per cent) of the wild type males could do so. From the other backcross the difference between the inseminating ability of the males was less clear cut, but the proportion successful was 6 per cent in ocra and 36 per cent in wild type. On the null hypothesis of no involvement of the X-chromosome in the causation of the male sterility, the proportion of inseminating males would not vary between the ocra and wild type segregants from the backcrosses. This null hypothesis is decisively rejected for both the backcross to morsitans $\left(\chi_{1}^{2}=\right.$ $43 \cdot 2, P<0 \cdot 001)$ and for that for centralis $\left(\chi_{1}^{2}=8 \cdot 6, P<0 \cdot 01\right)$.

\section{Discussion}

The data reported involve a centralis stock from the same area as that used by Curtis (1972) but collected about eight years later. The morsitans stock was wild type in the earlier work but mutant in the present case. Despite these differences the present work agrees with the earlier work in all respects where comparisons can be made, i.e., (i) the fecundity was much lower in the centralis $+\times$ morsitans $\delta$ cross than in the reciprocal; (ii) the fecundity of the backcrosses of the $F_{1}$ females was lower than that of the morsitans $q \times$ centralis $\delta$ crosses from which they had been produced; (iii) all $F_{1}$ males from both reciprocal crosses were unable to inseminate; (iv) the overall proportion of inseminating males from all the first backcrosses involving centralis and morsitans did not differ significantly between the earlier and the present work $\left(\chi^{2}=0 \cdot 27\right)$.

Use of the ocra gene to follow the inheritance of the morsitans $\mathrm{X}$ chromosome gave clear evidence that the X-chromosome was an important element in an interacting system which causes the male sterility. With 
regard to the other component(s) of the system, the results from the backcross to morsitans suggest that the $\mathrm{Y}$-chromosome is involved, i.e., the combinations of the morsitans $\mathrm{Y}$ with $\mathrm{X}$-chromosomes from the same or different sub-species yield a compatible or incompatible combination respectively. The exceptions (i.e., the sterile ocra males and the single fertile wild type male) might possibly result from crossing-over between the $\mathrm{X}$-chromosomes in the $\mathrm{F}_{1}$ females so that the ocra gene does not always remain associated with the morsitans male fertility factor(s). However, it is not known whether crossing-over can occur between the X-chromosomes of different tsetse sub-species; it does not occur between the X-chromosomes of different An. gambiae sibling species (Curtis and Chalkley, 1979). Now that a second X-chromosome marker (salmon eye) has been found in $G$. morsitans (Gooding, 1979), it would be possible to investigate crossing-over in sub-species hybrids.

In the case of the backcross to centralis, there was again evidence for a significant effect of the $\mathrm{X}$-chromosome but the fertility of the wild type segregants was much less than would be expected on a simple hypothesis of $\mathrm{X}-\mathrm{Y}$ interaction being the cause of male hybrid sterility. We suggest that autosomal factors must be involved here, an average of 25 per cent morsitans autosomal genes being sufficient in many cases to produce an adverse interaction with the centralis $\mathrm{X}$-chromosome.

The more important role of the $\mathrm{X}$ - than the $\mathrm{Y}$-chromosome in causing the hybrid male sterility would not be expected from the cytogenetic studies of Southern and Pell (1973), which showed that the sub-species have visible differences between their Y-chromosomes, but not between their Xchromosomes.

The backcross results showing a strong influence of the $\mathrm{X}$-chromosome, but also showing more sterility than would be expected on a simple $\mathrm{X}-\mathrm{Y}$ interaction hypothesis, closely resemble those obtained in An. gambiae crosses (Curtis, 1978 $a$ and $b$; Curtis and Sawyer, in preparation). The results suggest that interaction of the hemizygous $\mathrm{X}$-chromosome with alien $\mathrm{Y}$ and autosomal factors is the basis of Haldane's rule, i.e., that when only one sex of hybrid is sterile it is the heterogametic sex.

Recent field trials involving genetic control of tsetse flies have used radiation sterilisation (Dame et al., 1980; Cuisance et al., 1978). This has several advantages but the capital cost of radiation sources is considerable and it is possible that sterile males with better competitiveness could be produced if the sterile hybridization concept for tsetse control were revived. If barriers to cross mating exist between the $G$. morsitans sub-species (which are almost completely allopatric), $F_{1}$ hybrid males might be ineffective in the field. It seems likely that males such as the wild type products of the backcross to morsitans (fig. 1) would have behaviour compatible with that of morsitans females in the field because their genetic composition is largely of morsitans origin, apart from the centralis X-chromosome which makes them sterile. It is unlikely that such males could be produced economically from a segregating system such as that shown in fig. 1. It may be feasible, however, by crossing appropriate products of the two backcrosses to produce a fertile strain with its $\mathrm{X}$ - and $\mathrm{Y}$-chromosome of the same origin but its autosomes of mixed origin. Crossing this strain to one of the pure sub-species would be expected to generate sterile males with their genetic composition largely that of one of the pure sub-species. Non-inseminating males can partially 
deter their mates from re-mating in confined conditions in the laboratory (Curtis, 1972); it remains to be seen whether they would do so more effectively under natural conditions. This ability would be essential if hybrid males are to have any prospects as a tsetse control agent.

Acknowledgements. - We are gratefui to Dr A. M. Jordan for his interest in this project, which was conducted at the Tsetse Research Laboratory, University of Bristol, to Dr Helle, University of Amsterdam for providing the "ocra" mutant stock of G. m. morsitans and to DrS. K. Moloo, ILRAD, Nairobi, for the supply of G. m. centralis. The financial support of the Overseas Development Administration of the U.K. Government is gratefully acknowledged.

\section{REFERENCES}

BOLLAND, H. R., VAN BUREN, A., VAN DER GEEST, C. P. S., AND HELle, W. 1974. Marker mutations in the tsetse fly Glossina morsitans. Entomologia experimentalis et applicata, 17, 522-524.

CUISANCE, D., POliTZAR, H., ClAIR, M., SELlin, E., AND TAZE, Y. 1978. Impact des lâchers de mâles stériles sur les niveaux de deux populations sauvages de Glossina palpalis gambiensis en Haute-Volta (Sources de la Volta Noire). Rev. Elev. Méd. vét. Pays trop., 31, 315-328.

CURTIS, C. F. 1972. Sterility from crosses between sub-species of the tsetse fly Glossina morsitans. Acta tropica, 29, 250-268.

CURTIS, C. F. 1978a. Hybrid sterility in the Anopheles gambiae complex: mechanism and possible means of using it for genetic control (Abstract). Medical Entomology Centenary, Symposium Proceedings, Royal Society of Tropical Medicine and Hygiene, p. 133.

CURTIS, C. F. $1978 b$. Manipulation of the sterility system in sibling species crosses within the Anopheles gambiae complex (Abstract). Heredity, 40, 327-328.

CURTIS, C. F., AND CHALKLEY, J. 1979. Lack of recombination between the X chromosomes of different members of the Anopheles gambiae complex. Heredity, 42, 323-326.

DAME, D. A., WILliaMSON, D. L., COBB, P. E., GATES, D. B., WARNER, P. V., MTUYA, A. G., AND BAUMGARTNER, H. 1980. Integration of sterile insects and pesticides for the control of tsetse fly. Isotope and Radiation Research on Animal Diseases and their Vectors. International Atomic Energy Agency, Vienna, pp. 267-280.

FRACCARO, M., TIEPOLO, L., LAUDANI, U., MARCHI, A., AND JAYAKAR, S. N. 1977. Y chromosome controls mating behaviour of Anopheles mosquitoes. Nature, 265, 327328.

GOODING, R. H. 1979. Genetics of Glossina morsitans morsitans (Diptera: Glossinidae). III. Salmon, a sex-linked, maternally influenced, semi-lethal eye color mutant. Canadian Entomologist, 3, 557-560.

JACKSON, C. H. N. 1945. Pairing of Glossina morsitans with G. swynnertoni. Proceedings of the Royal Entomological Society of London (A), 20, 106.

MACHADO, A. DE B. 1970. Les races géographiques de Glossina morsitans. Proceedings of the 1st Symposium on Tsetse Fly Breeding under Laboratory Conditions, Lisbon, 1969, 471 486.

MEWS, A. R., LANGLEY, P. A., PIMLEY, R. W., AND FLOOD. M. E. T. 1977. Large-scale rearing of tsetse flies (Glossina spp.) in the absence of a living host. Bull. ent. Res. 67, 119-128.

POTTS, W. H. 1944. Tsetse hybrids. Nature, 154, 606-607.

SOUTHERN, D. I., AND PELL, P. E. 1973. Chromosome relationships and meiotic mechanisms of certain morsitans group tsetse flies and their hybrids. Chromosoma (Berlin), 44, 319-334.

VANDERPLANK, F. L. 1944. Hybridization between Glossina species and suggested new method for control of certain species of tsetse. Nature, 154, 607-608.

VANDERPLANK, F. L. 1947. Experiments on hybridization of tsetse flies and the possibility of a new method of control. Transactions of the Royal Entomological Society of London, 98 , $1-18$.

VANDERPLANK, F. L. 1948. Experiments on cross breeding tsetse flies. Annals of Tropical Medicine and Parasitology, 42, 135-152.

VANDERPLANK, F. L. 1949. The classification of Glossina morsitans, including a description of a new subspecies, varieties and hybrids. Proceedings of the Royal Entomological Society of London (B), 18, 56-64. 